holds with $s=2.81 \pm 0.13$, which is, within experimental errors, almost the same as that for the photons. It was also found that in the experiment of Series 1 (with shielding $S$ in position) photon- and electron-initiated showers occur at the same absolute rate within 10 percent at energies $0.3-2.0 \mathrm{Bev}$. In Series 2, there were about 30 percent more photon-initiated than electroninitiated showers observed. This is probably essentially because the side counters $E$ excluded electrons with good efficiency, whereas photons from the sides were registered by the apparatus.

The interpretation of the results at energies lower than $0.2 \mathrm{Bev}$ is complicated because the arrangement used was not very sensitive in this region. A more elaborate apparatus is now being constructed with the purpose of carrying out energy measurements of high energy photons and electrons with increased precision.

\section{CONCLUSION}

The results of this work show that a suitable scintillation counter arrangement making use of large plastic scintillators provides a new method of measuring the energies of high energy photons or electrons, or the energy distribution of a spectrum of high energy photons or electrons with satisfactory precision. The energy spectra of cosmic-ray photons and electrons at ground level obtained by this method are not inconsistent with previous results obtained by others. ${ }^{1-6}$

The author wishes to express his hearty thanks to Professor Marcel Schein for many valuable discussions and for the privilege of working in the Cosmic Ray Laboratory at Chicago. He also thanks Mr. T. Bowen for numerous constructive discussions and for his valuable cooperation.

\title{
On the Damping of Virtual Nucleon-Pair Formation in Pseudoscalar Meson Theory*
}

\author{
K. A. BrueckNer, Indiana University, Bloomington, Indiana \\ AND \\ M. Gell-Mann ANd M. Goldberger, University of Chicago, Chicago, Illinois, \\ (Received January 15, 1953)
}

\begin{abstract}
The modifications of the propagation characteristics of a nucleon which result from the presence of a strongly coupled mesonic self-field are estimated from the consideration of a simple subset of radiative corrections to the nucleon propagation function. It is found that reactive effects markedly inhibit nucleon pair formation so that the contributions from the pseudoscalar coupling term which do not involve nucleon pairs are strongly enhanced relative to those involving pair formation. In addition, the meson pair coupling term, which results from nonrelativistic approximations to the relativistic linear coupling term and is intimately connected with nucleon pair formation, is strongly damped. The relation of this result to the nonrelativistic theory of Wentzel is discussed.
\end{abstract}

$\mathrm{O}^{\mathrm{s}}$ NE of the most characteristic features of pseudoscalar meson theory with pseudoscalar coupling is the large value of the matrix elements of the interaction for processes in which a nucleon pair is produced. This effect is usually stated qualitatively by noting that the operator $\gamma_{5}$ has matrix elements of the order of $v / c$ ( $v$ the nucleon velocity) for processes in which no nucleon pairs are produced but of the order of unity for nucleon pair production. The consequences of this effect are well known for a number of important phenomena, particularly (1) meson-nucleon scattering, where the contribution to the scattering involving nucleon pair production in the intermediate states gives rise in the weak coupling limit to very large $s$ wave scattering, ${ }^{1}$ and (2) nuclear forces where the fourthorder potential ${ }^{2}$ arises almost entirely from nucleon pair formation in the intermediate states.

${ }^{*}$ This work was supported in part by a grant from the National Science Foundation.

1 Ashkin, Simon, and Marshak, Progr. Theoret. Phys. (Japan) $5,634(1950)$.

2 K. M. Watson and J. V. Lepore, Phys. Rev. 76, 1157 (1949); H. A. Bethe, Phys. Rev. 76, 191 (1949); M. Lévy, Phys. Rev. 86, 806 (1952); J. V. Lepore, Phys. Rev. 88, 750 (1952).
A convenient method which exhibits these features of the theory is that given by the transformations of Dyson $^{3}$ and of Foldy, ${ }^{4}$ which show that the pseudoscalar coupling term

$$
H_{p s}=i g \bar{\psi} \gamma_{5} \tau_{\alpha} \psi \varphi_{\alpha}
$$

may be transformed into an expression nonlinear in the meson field variables, which in the nonrelativistic limit has as leading terms in $g$,

$$
(g / 2 M) \boldsymbol{\sigma} \cdot \boldsymbol{\nabla}(\tau \cdot \varphi) \rho(r)+\left(g^{2} / 2 M\right) \varphi^{2} \rho(r),
$$

where $\rho(r)$ is the nucleon source density. The first term is the usual pseudovector coupling term; the second is a meson pair coupling which is very closely associated with nucleon pair formation, arising as it does from the pairing of two pseudoscalar interaction terms $\mathrm{H}_{p s}$ in which the two pairs of nucleon operators create or annihilate a nucleon pair with the result that the native elements of the two $\gamma_{5}$ operators are of the order of unity. This meson-pair term gives rise to large $s$ wave

\footnotetext{
${ }^{3}$ F. J. Dyson, Phys. Rev. 73, 929 (1948)
}

${ }^{4}$ L. L. Foldy, Phys. Rev. 84, 168 (1951). 
meson scattering (in Born approximation) and to the dominant fourth-order potential in nuclear forces.

It has been shown by Wentzel ${ }^{5}$ that if only the pair term is considered, exact solutions can be obtained to both the meson-nucleon scattering and the nuclear force problems (at distances much greater than the cut-off radius introduced to make the theory finite). These results are closely related to the perturbation theory results except that the coupling parameter $\mathrm{g}^{2}$ is replaced by

$$
g^{2}\left[1+\left(g^{2} / 4 \pi\right) A / M\right]^{-1},
$$

where $A$ is a cut-off momentum characteristic of the nonrelativistic theory considered by Wentzel. ${ }^{6}$ Since it is expected in this theory that $A$ will be approximately equal to $M$, and, in addition, since $g^{2} / 4 \pi$ is known from experiment to be of the order of 10 to 40 , it is apparent that both the meson scattering and the fourthorder nuclear potential (both proportional to $\mathrm{g}^{4}$ ) will be depressed by at least two orders of magnitude relative to the perturbation result. This result is somewhat difficult to interpret in terms of the original relativistic theory, since it is apparent that an undetermined amount of renormalization effects are included in the nonrelativistic theory of Wentzel.

If, however, we return to the original untransformed form of the relativistic theory, we can easily show that Wentzel's result is essentially correct, even if renormalization effects are properly taken into account. We consider specifically the radiative corrections (see Fig. 1) of a particularly simple sort to the $S_{F}$ function of Feynman (the nucleon propagation function). The higher order effects which arise from the repeated emission and reabsorption of a meson by the nucleon can equally well be considered to be the repeated scattering of a virtual meson by the nucleon. The largest contribution to this process comes from the repeated creation and annihilation of nucleon pairs at successive meson vertices; accordingly, the iteration of this particular type of diagram is very closely related to the iteration of the meson-pair term in the nonrelativistic approximation to the coupling [Eq. (2)].

The correction of order $g^{2}$ to the $S_{F}$ function is [with $S_{F}$ in momentum space equal to $-i(\mathbf{P}-i M)^{-1}$ and $\left.\mathbf{P}=P_{\mu} \gamma_{\mu}\right]$

$$
\begin{aligned}
-S_{F}\left(P_{\mu}\right) \frac{g^{2}}{(2 \pi)^{4}} \int d^{4} k \gamma_{5} \tau_{i} S_{F} & \left(P_{\mu}-k_{\mu}\right) \\
& \times\left(k^{2}+\mu^{2}\right)^{-1} \gamma_{5} \tau_{i} S_{F}\left(P_{\mu}\right) .
\end{aligned}
$$

The evaluation of this expression and the renormalization of mass and charge are easily done using the methods of Dyson ${ }^{7}$ the $S_{F}$ function corrected for the first-

\footnotetext{
${ }^{5}$ G. Wentzel, Phys. Rev, 86, 802 (1952)

${ }^{6}$ S. D. Drell and E. M. Henley [Phys. Rev. 88, 1053 (1952)] have also noted that the higher order effects strongly depress the effective meson pair coupling. Their nonrelativistic treatment, however, does not properly treat the renormalization effects.

${ }^{7}$ F. J. Dyson, Phys. Rev. 75, 1736 (1949).
}

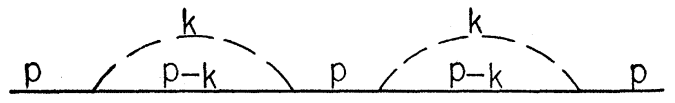

FIG. 1. Radiative corrections to the $S_{F}$ function. The subset to be considered consists of the iteration of this particular correction. The solid line represents a nucleon, the dashed line a meson.

order radiative effect is

$$
S_{F}\left(P_{\mu}\right)\left[1-\left(3 g^{2} / 16 \pi^{2}\right) f\left(P_{\mu}\right)\right]
$$

where

$$
\begin{array}{r}
f\left(P_{\mu}\right)=\int_{0}^{1} d x\left[-\frac{(1-x) \mathbf{P}+i M}{\mathbf{P}-i M} \ln \frac{\phi\left(P^{2}\right)}{\phi\left(-M^{2}\right)}\right. \\
\left.+\frac{2 M^{2} x^{2}(1-x)}{\phi\left(-M^{2}\right)}\right]
\end{array}
$$

and

$$
\phi\left(P^{2}\right)=(1-x) \mu^{2}+x M^{2}+x(1-x) P^{2} .
$$

The correction term $f\left(P_{\mu}\right)$ vanishes, if the nucleon 4-momentum $P_{\mu}$ is that of a free particle, i.e., if $P_{\mu}=i M$, $P^{2}=-M^{2}$, this feature being the consequence of the renormalization.

This result is easily extended to an interation of the simple series of radiative corrections we wish to consider; the series obtained is

$$
\begin{aligned}
S_{F}^{\prime}\left(P_{\mu}\right) & =S_{F}\left(P_{\mu}\right) \sum_{n=0}^{\infty}\left[\left(-3 g^{2} / 16 \pi^{2}\right) f\left(P_{\mu}\right)\right]^{n} \\
& =S_{F}\left(P_{\mu}\right)\left[1+\left(3 g^{2} / 16 \pi^{2}\right) f\left(P_{\mu}\right)\right]^{-1}
\end{aligned}
$$

Some qualitative features of this correction factor are easily obtained. If the nucleon 4-momentum vector $P_{\mu}$ is close to that of a free particle, then $f\left(P_{\mu}\right)$ is small, being of the order of $\left(P^{2}+M^{2}\right) / M^{2}$. For example, if a nonrelativistic approximation to the matrix elements of $\gamma_{5}$ is used, ${ }^{8}$ then pair production is absent and $\left(P^{2}+M^{2}\right) / M^{2}$ differs from zero only by terms of the order of $\Delta P^{2} / M^{2}$, where $\Delta P^{2}$ measures the degree to which the virtual nucleon is not propagating as a free particle. In a nuclear force problem, this will be of the order of the ratio of the potential strength to the nucleon rest mass and accordingly small if the two nucleon separation is such that the motion of the nucleons is nonrelativistic. We can therefore conclude that such corrections are small, if the motion of the nucleons (including virtual states) is predominantly nonrelativistic. In meson-nucleon scattering, if the intermediate states do not involve nucleon-pair production (as is typical of $p$ wave scattering via the gradient interaction), the radiative effects will similarly be small. The absence of strong damping from radiative effects in such processes is closely related to the lack of violent alterations of the self-field of the nucleon when only small momentum changes in its motion occur. The

${ }^{8}$ This approximation leads to the replacement of $\gamma_{5}$ by $\frac{1}{2} \sigma \cdot \Delta \mathbf{P} / M$, where $\Delta \mathbf{P}$ is the momentum transfer to the meson at the vertex, i.e., the change in the nucleon momentum. 
effects of the renormalization are clearly evident here since the strong damping effects would otherwise occur even if the virtual nucleons were nonrelativistic and no pairs were formed; i.e., the nonrelativistic gradienttype coupling would also be strongly damped.

The true relativistic features of pseudoscalar theory are, however, affected in a qualitatively different manner. If the propagation function $S_{F}{ }^{\prime}\left(P_{\mu}\right)$ appears between two meson vertices, then since the matrix elements of $\gamma_{5}$ are large for pair annihilation and creation at the vertices, the 4-momentum $P_{\mu}$ which appears in the $S_{F}{ }^{\prime}$ function is predominantly that of the antinucleon. For a simple double vertex the matrix element is

$$
g^{2} \bar{\psi} \tau \cdot \varphi \gamma_{5} S_{F}^{\prime}\left(P_{\mu}\right) \tau \cdot \varphi \gamma_{5} \psi
$$

From the anticommutation of $\gamma_{5}$ with $\gamma_{\mu}$, it follows that this expression can also be written as

$$
g^{2} \bar{\psi} \tau \cdot \varphi \gamma_{5} S_{F}\left(P_{\mu}\right) \tau \cdot \varphi \gamma_{5}\left[1+\frac{3 g^{2}}{16 \pi^{2}} f\left(-P_{\mu}\right)\right]^{-1} \psi,
$$

if we make use of Eq. (8) for $S_{F}{ }^{\prime}$. The change in sign of the 4-momentum $P_{\mu}$ in $f\left(P_{\mu}\right)$ radically changes the size of the correction terms; if we again consider nonrelativistic 4 momenta in the sense that $P^{2} \approx-M^{2}$, then

$$
f\left(-P_{\mu}\right)=1+O\left(\left(P^{2}+M^{2}\right) / M^{2}\right) .
$$

Accordingly the radiative effects ${ }^{9}$ give approximately a simple correction to the coupling constant, i.e., Eq. (10) becomes

$$
g^{2} /\left(1+3 g^{2} / 16 \pi^{2}\right) \bar{\psi} \tau \cdot \varphi \gamma_{5} S_{F}\left(P_{\mu}\right) \tau \cdot \varphi \gamma_{5} \psi
$$

It must be emphasized that this very marked depression in the effective coupling results only if pair formation dominates the virtual nucleon states.

The matrix element of Eq. (11) takes on a simple form if its nonrelativistic limit is taken; in this case $\mathbf{P} \approx i M$, and

$$
g^{2} \bar{\psi}_{\tau} \cdot \varphi \gamma_{5} S_{F}^{\prime} \tau \cdot \varphi \gamma_{5} \psi \approx g^{2} /\left(1+3 g^{2} / 16 \pi^{2}\right) \frac{\varphi^{2}}{2 M} \rho(r),
$$

which is the pair coupling term resulting characteristically from the Dyson-Foldy transformations except for the decrease in the strength of the coupling. This depression in the meson pair coupling is of the same form as that found by Wentzel (except for a numerical factor); its effect is to reduce the effective strength of the pair coupling by roughly a factor of ten.

The physical interpretation of this result is clear; the

${ }^{9}$ M. Ruderman (University of California Radiation Laboratory Report UCRL report 1876, July 1,1952) has evaluated the lowest order radiative correction to the fourth-order potential corresponding to a correction to the $S_{F}$ function and finds that the sixth-order potential is of the same magnitude as the fourth-order but is repulsive; this is in agreement with our conclusions if the correction term of Eq. (12) to the $S_{F}$ function is expanded and the lowest order term is kept. nucleon pair production via the $\gamma_{5}$ coupling leads to violent perturbations of the self-field of the nucleon; the different features of the fields of a nucleon and antinucleon lead (when the coupling is strong) to strong radiative effects which markedly inhibit nucleon-antinucleon transitions (or reversal of the direction of motion of the nucleon in time, in the Feynman sense). This "inertial" effect has also been noted by $\mathrm{Hu}^{10}$ in his considerations of pseudoscalar theory with pseudovector coupling; he found that radiative effects associated with the nucleonic self-field of a meson inhibit the emission of high energy virtual mesons and, accordingly, remove the divergences of the theory which result from the derivative coupling. It would appear that similar "inertial" effects would be expected to appear in any strong coupling theory (strongly coupled because of a large coupling constant or because of a divergent interaction) to modify markedly the results of a straightforward perturbation expansion.

The damping of the meson pair coupling (resulting from the damping of nucleon-pair production) will lead to the consequences already pointed out by Wentzel, ${ }^{5}$ i.e., to rather weak $s$ state meson scattering and to the near elimination of that part of the fourth-order potential which arises from the meson pair terms. The contributions from the nonrelativistic part of the coupling, namely, the linear pseudovector coupling term (which is almost undamped for nonrelativistic nucleon velocities by the radiative corrections), will be accordingly strongly enhanced relative to the pair coupling and can, in fact, be expected to dominate mesonic phenomena at low energy.

Similar results for the case of meson scattering by nucleons have already been found on the basis of a rather different argument by $\mathrm{Chew}^{11}$ and Dyson. ${ }^{12}$ They found that the meson-pair term derived from pseudoscalar theory was very strongly damped in the scattering problem when correctly treated, since it gives rise to a meson-nucleon potential of short range which is strongly repulsive and accordingly scatters rather weakly. This prediction is supported by experiment which shows relatively weak $s$ wave scattering. ${ }^{13}$ From the above analysis it is apparent that this feature of the theory results more generally from the damping caused by radiative reactive effects.

In conclusion, we wish to remark that although the effects we have discussed arise from a particularly simple subset of higher order corrections to the $S_{F}$ function, it seems probable that the qualitative feature which results, namely, the strong reactive effects of the strongly coupled self field, will persist in a more complete analysis of the theory.

The author is indebted to Professor K. M. Watson for some valuable discussions.

${ }^{10}$ Ning Hu, Phys. Rev. 80, 1109 (1950).

"Geoffrey Chew (private communication).

12 F. J. Dyson (private communication)

${ }^{13}$ Anderson, Fermi, Nagle, and Yodh, Phys. Rev. 86, 793 (1952). 